\title{
THE TOXIC FACTORS IN EXPERIMENTAL TRAUMATIC SHOCK. III. SHOCK ACCOMPANYING MUSCLE ISCHEMIA AND LOSS OF VASCULAR FLUID ${ }^{1}$
}

\author{
By S. S. KETY, I. T. NATHANSON, A. L. NUTT, A. POPE, P. C. ZAMECNIK, \\ J. C. AUB, AND A. M. BRUES \\ (From the Medical Laboratories of the Collis P. Huntington Memorial Hospital of \\ Harvard University at the Massachusetts General Hospital, Boston, Massachusetts)
}

(Received for publication April 9, 1945)

A discussion of the hemodynamic factors involved in the production of traumatic shock has already been presented (24). It was concluded that on the basis of both clinical and experimental observations, local loss of vascular fluid into the traumatized areas must be considered the principal cause for the development of shock following injuries, but that other factors such as nociceptive nervous impulses and hypothetical toxins elaborated in the damaged tissue have not been ruled out as contributory elements in this process. This paper will be concerned with describing a further attempt to demonstrate the action of a toxic factor by the use of the triceps surae ligation procedure previously described (14).

The theory of traumatic toxemia has been based upon two types of experimental studies. One of these has consisted of the parenteral administration to normal animals of various extracts and products of both normal and damaged tissues with the development of a shock-like state in the recipient. The significance of experiments of this sort will be discussed subsequently (25).

A more direct approach to the problem consists in producing shock in animals by actual traumatizing procedures of such a nature as to permit measurement or control of other factors (fluid loss, neurogenic) and hence an evaluation of the possible etiologic rôle of toxins. Pioneer studies of this sort were carried out during the first World War $(26,27,1)$. The findings were interpreted as indicating the operation of a humoral agent in the pathogenesis of shock and resulted at the time in a widespread acceptance of the theory of

1 This is reprint No. 607 of the Cancer Commission of Harvard University.

The work described in this paper was done under a contract, recommended by the Committee on Medical Research, between the Office of Scientific Research and Development and the Massachusetts General Hospital. traumatic toxemia. These conclusions, however, have since been considered as inconclusive because of incomplete estimation of the local fluid loss which probably occurred in their animals (28 to 31).

Recently, traumatic shock was induced in dogs by repeated hammering of an extremity, and local fluid loss was measured by a water displacement technique (32). They concluded that although local fluid loss was a major etiological factor, it alone in their experiments was insufficient to produce fatal shock. In their series of 20 dogs, however, the average local fluid loss was 3.4 per cent of the body weight and 80 per cent of the animals exhibited a local fluid loss of over 3 per cent of the body weight. These figures are of the same order of magnitude as the amounts of simple hemorrhage which most investigators find necessary to produce shock (33 to 41 ). Consideration should also be given to the fact that their measurements of effusion gave minimal values and that plasma loss might be more detrimental than loss of similar quantities of whole blood.

Some investigators have attempted to demonstrate the operation of toxic factors by cross circulation experiments of varying degrees of complexity (42 to 44). Although it is likely that toxic factors, if present, might exert some effect on the untraumatized animal of a cross-circulated pair, it is obvious that this animal would lose vascular fluid into the traumatized limb of the other. It is surprising that positive results with such experiments were not more frequent. Recognizing this difficulty, two groups have attempted to avoid it by substituting for cross circulation, repeated and equal cross transfusions of whole blood. The continuous exchange of equal quantities of blood between a traumatized and a normal dog with the occasional development of shock in 
the latter was interpreted as evidence for the action of a toxic factor arising in the traumatized region of the former (32). Since the local fluid loss is almost entirely plasma, while the cross transfusions are equivalent only in terms of whole blood, it is apparent that in these experiments as in the simple cross circulation experiments the normal animal continues to lose plasma into the traumatized limb of the other. Shock produced in normal dogs by cross transfusion with a heartlung traumatized limb preparation was assumed to be due to the action of a toxin (45). In each of the four experiments reported there was a local loss of fluid of about one liter into the traumatized limb. How much of this came from the normal dog it is difficult to judge, since the authors fail to state the volume of blood in their reservoir. It could, however, have represented an appreciable plasma loss from this animal if the hematocrit of the blood infused into the latter was higher than that of the blood simultaneously withdrawn. Two things should be noted regarding these experiments; in many cases blood may have been traumatized, and in the cases cited the results of experiments have been variable.

Using a bandaging technique to minimize local fluid loss on sympathectomized and spinal animals, it has been concluded that there was evidence for the operation of some factor other than local fluid loss (46 to 48). Quite recently evidence has been presented that trauma plus ischemia, but not trauma alone, results in shock even when the reduction in blood volume incident to the local fluid loss is corrected continuously (4). Although no conclusion was drawn regarding toxic factors, it would be important to rule out possible neurogenic elements before these experiments are regarded as the good evidence which they appear to be for the operation of the products of damaged and ischemic tissues in the production of this type of shock. In summary, the evidence for the toxemic theory of shock, while suggestive, is based upon experiments where it has been difficult to control other causative mechanisms.

Nor is the evidence against the toxemic theory conclusive. Experiments in which local fluid loss is measured and found to be sufficient to cause shock $(28,29,31,49$ to 51$)$ were of great value in establishing the importance of this process in the pathogenesis of traumatic shock but do not rule out the possible contributory rôle of other factors. It has been shown that when local fluid loss in regions of trauma is artificially restricted, shock does not occur $(3,4,46)$. Although such experiments speak against the likelihood that factors other than fluid loss can cause shock, they do not rule out the possibility that such factors may facilitate or aggravate the effects of reduced blood volume. It is conceivable that there may be in traumatic shock certain humoral substances released from areas of tissue damage which have no specific effect on the intact circulatory system and could not in themselves cause shock or even a fall in blood pressure, yet by virtue of their effects on cell metabolism could play a rôle in lowering the resistance to blood loss or anoxia. Experiments in the past have not been designed either to establish or disprove the existence of such factors and for that reason the present work was undertaken. A localized area of tissue damage was produced in a series of dogs, and these animals together with a group of untraumatized controls were subjected to controlled hemorrhage. It was felt that by comparing the incidence of shock in the two groups, the possible rôle of factors other than vascular fluid loss could be evaluated.

\section{METHODS}

Mongrel street dogs were used in all experiments. They were anesthetized with sodium pentobarbital in an initial dose of $30 \mathrm{mgm}$. per $\mathrm{kgm}$. of body weight and subsequent doses of $30 \mathrm{mgm}$. as required. The muscle ligations were performed according to the procedure described in the first paper of this series (14) but without sterile precautions. Rubber ligatures were kept in place for 5 hours and then released. During this interval the animals were bled from a catheter inserted through the external jugular vein into the right auricle, in steps of 1 or 0.5 per cent of body weight at about 30 -minute intervals until the total bleeding reached a predetermined amount from 0 to 3 per cent of body weight. Control animals were not operated upon but were bled corresponding amounts at the same time.

Blood pressures were taken at frequent intervals by means of a needle inserted into the femoral artery and connected to a mercury manometer. Determinations of venous oxygen saturations and hemoglobin concentrations were made on mixed venous blood obtained from the right auricle. Blood loss incidental to the operation and to the blood pressure measurements was estimated by saving all sponges used and determining the hemoglobin content of an aqueous extract.

The release of the muscle ligature is immediately followed by a progressive swelling of the limb which begins 
in the gastrocnemius and spreads throughout the leg from the thigh to the ankle. In some dogs an attempt was made to measure the amount of this local fluid loss in the following manner.

On the medial aspect of the closely-shaved leg an ink line is drawn longitudinally bisecting this surface from the groin to the internal malleolus (Figure 1). At 8 equidistant points along this line, and in each case perpendicular to it, circumferences are drawn about the limb. By treating the segment of limb between the 2 extreme

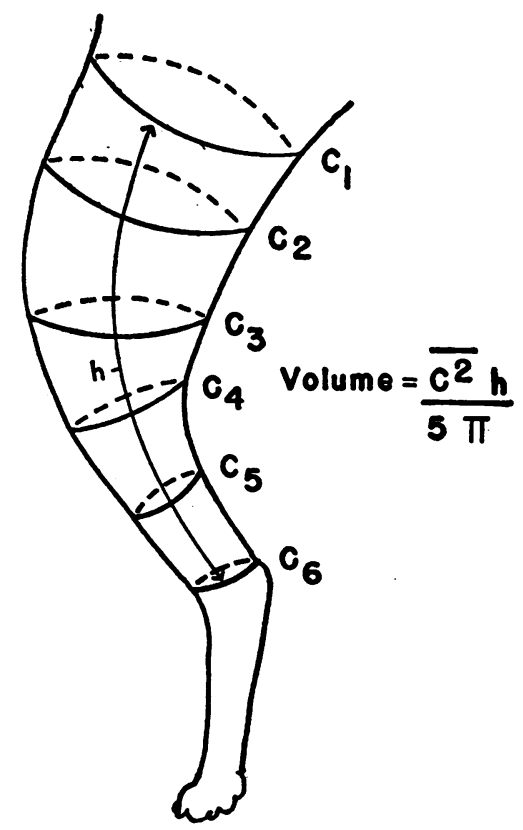

Fig. 1. Illustration of the Method Used in CalcuLating Fluid Loss into a Limb

circumferences as a flexed, truncated, elliptical cone with 1 semi-axis twice the length of the other (an assumption which can be shown to fit the actual shape remarkably closely), it is possible to derive the volume of this segment in terms of the length of the longitudinal axis (h) and the mean of the squares of the several circumferences $\left(\overline{c^{2}}\right)$ thus :

$$
\mathrm{V}=\frac{\overline{c^{2} h}}{5 \pi}
$$

Calculation of the volume of a limb segment obtained by this method agreed within 2 per cent of that observed by water displacement. Measurement of the increase in volume of the limb by this technique is, however, minimal since only a large segment of the leg is included, and it is known that such fluid dissects its way not only throughout the limb but even into the flanks. To test the recovery of added fluid by this technique, known quantities of plasma were injected into the limb of a dead dog and the increase in volume determined. There was a consistent recovery of only 80 per cent of the injected fluid, the remainder having spread outside the area of measurement. Values for volume increase obtained by this tech- nique have, therefore, been corrected by a factor of 1.25 in an attempt to approximate the true magnitude of the effusion. This technique was used because it permits frequent determinations of local fluid loss throughout the course of the experiment.

The animals operated upon were followed for at least 5 hours after the release of the ties and the controls for a corresponding length of time.

\section{RESULTS AND DISCUSSION}

The experimental data and results are presented in Tables I and II. The last column indicates the development or absence of shock, the criteria for which included a progressive decline of blood pressure to below $70 \mathrm{~mm}$. $\mathrm{Hg}$, and (where this was measured) a consistently depressed mixed venous oxygen saturation.

There were 41 dogs with muscle ties plus hemorrhage and 23 dogs with hemorrhage alone. The operative blood loss was measured in 23 of the dogs operated upon and 11 controls which were only bled. The average operative blood loss in the 23 operated dogs amounted to 0.5 per cent of the body weight, and this figure was used in estimating the total blood loss of those dogs in which the operative loss was not directly determined. Similarly, the average incidental blood loss in the 11 dogs with hemorrhage alone amounted to 0.2 per cent of body weight and this was added to the purposeful blood loss of other such animals in which it was not measured.

The effusion into the operated limbs was measured in 7 dogs. In these dogs the measured effusion was multiplied by 1.25 to obtain an estimate of the total effusion as described above. The average measured effusion was 1.3 per cent \pm 0.2 per cent of the body weight, and this figure multiplied by 1.25 , or 1.6 per cent, was used in estimating the total fluid loss in those dogs in which the effusion was not directly measured.

The calculation gives a value for the effusion which is almost certainly minimal. Any fluid dissecting its way superiorly into the flank or along the back was not measured by the technique employed. This has been by no means a negligible amount in similar experiments $(30,52)$. In addition, not inconsiderable amounts of unmeasured fluid dissected downward into the ankles which exhibited marked pitting edema in almost all instances. Moreover, no attempt has been made to estimate the equivalence of this traumatic 
TABLE I

Observations on dogs with muscle-tie and graded hemorrhage

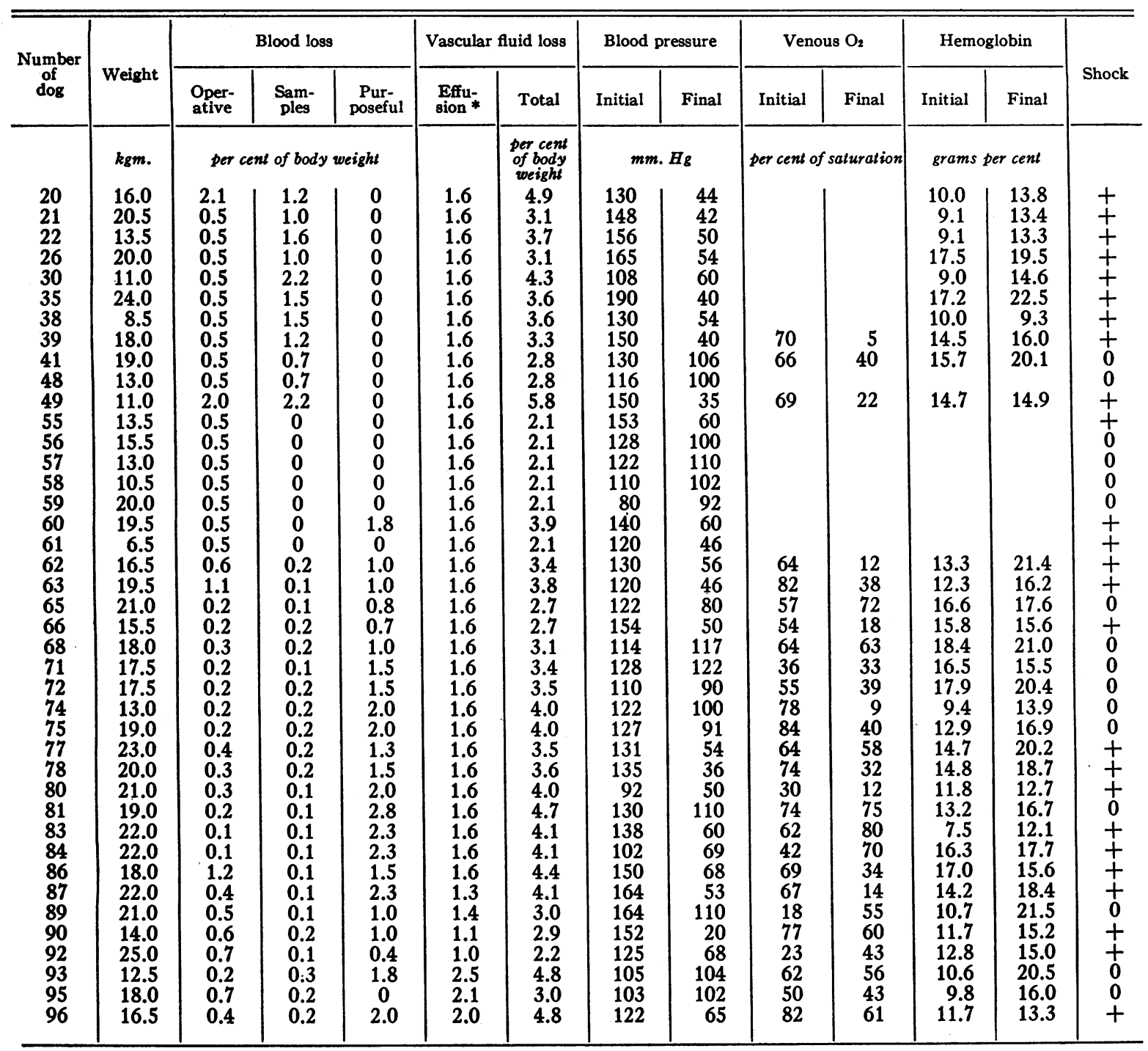

* Effusion was actually measured in numbers 87 to 96 . Figures in other cases are estimated (see text).

effusion in terms of whole blood loss. The total protein of this effusion fluid has been found to average 4.2 grams per $100 \mathrm{ml}$. which is 70 per cent of the value of the corresponding plasma protein concentrations. It seems highly probable that the loss from the circulation of a given volume of plasma is more detrimental than that of the same volume of whole blood since the former contains all of the osmotically-active constituents of the latter, while the proportionally greater hemoconcentration may result in even further cir- culatory embarrassment because of the greater increase of blood viscosity (53).

The incidence of shock with graded amounts of total fluid loss in both the operated and control animals (Table III) demonstrates no significant difference between the two groups. These dogs were bled fixed amounts, and the incidence of shock at each degree of total fluid loss determined; whereas most investigators studying the effects of hemorrhage have bled dogs until they went into shock, a difference in technique which ought 
TOXIC FACTORS IN EXPERIMENTAL TRAUMATIC SHOCK. III

TABLE II

Observations on dogs with hemorrhage alone

\begin{tabular}{|c|c|c|c|c|c|c|c|c|c|c|c|c|}
\hline \multirow{2}{*}{$\begin{array}{l}\text { Number } \\
\text { of dog }\end{array}$} & \multirow{2}{*}{ Weight } & \multicolumn{4}{|c|}{ Blood loss } & \multicolumn{2}{|c|}{ Hemoglobin } & \multicolumn{2}{|c|}{ Venous $\mathrm{O}_{2}$} & \multicolumn{2}{|c|}{ Blood pressure } & \multirow{2}{*}{ Shock } \\
\hline & & Operative & Samples & Purposeful & Total & Initial & Final & Initial & Final & Initial & Final & \\
\hline & kgm. & \multicolumn{4}{|c|}{ per cent of body weight } & \multicolumn{2}{|c|}{ grams per cent } & \multicolumn{2}{|c|}{ per cent of saturation } & \multicolumn{2}{|c|}{$m m . H g$} & \\
\hline & 17.5 & 0.2 & & .6 & 4.8 & & & & & 104 & 34 & + \\
\hline 2 & 19.0 & 0.2 & & .1 & 2.3 & 15.4 & 15.4 & & & 95 & 20 & $t$ \\
\hline 3 & 17.5 & 0.2 & & & 4.6 & 11.0 & 9.9 & & & 120 & 30 & $\dot{t}$ \\
\hline 4 & 10.0 & 0.2 & & 8 & 5.0 & 11.6 & 5.5 & & & 110 & 46 & + \\
\hline 5 & 14.5 & 0.2 & & .9 & 6.1 & 14.3 & 9.0 & & & 120 & 28 & + \\
\hline 6 & 10.5 & 0.2 & & .0 & 5.2 & 17.4 & 16.0 & & & 120 & 36 & + \\
\hline 7 & 20.0 & 0.2 & & .7 & 5.9 & 12.3 & 15.1 & & & 118 & 56 & + \\
\hline $\begin{array}{l}8 \\
0\end{array}$ & 16.5 & 0.2 & & 9 & 6.1 & 9.7 & 13.8 & & & 128 & 36 & \pm \\
\hline $\begin{array}{r}9 \\
10\end{array}$ & $\begin{array}{l}15.5 \\
12.0\end{array}$ & 0.2 & 1.8 & 16 & 2.0 & 12.0 & 13.1 & & & 110 & 100 & 0 \\
\hline 12 & 22.5 & 0.2 & & .4 & $\begin{array}{l}3.0 \\
3.6\end{array}$ & 16.0 & $\begin{array}{l}12.4 \\
13.3\end{array}$ & & & 148 & 35 & $t$ \\
\hline 18 & 21.0 & 0.2 & 1.4 & 0 & $\begin{array}{l}.0 \\
1.6\end{array}$ & 12.7 & 17.4 & & & 160 & 138 & 0 \\
\hline 67 & 18.0 & 0.1 & 0.1 & 2.0 & 2.2 & 13.9 & 15.9 & 60 & 37 & 136 & 50 & + \\
\hline 70 & 17.5 & 0.1 & 0.2 & 1.0 & 1.3 & 16.2 & 20.2 & 64 & 37 & 128 & 132 & 0 \\
\hline 73 & 15.5 & 0.1 & 0.2 & 1.5 & 1.8 & 14.1 & 19.2 & 70 & 29 & 146 & 112 & $\mathbf{0}$ \\
\hline 76 & 21.0 & 0.2 & 0.2 & 2.0 & 2.4 & 11.2 & 14.6 & 84 & 42 & 150 & 125 & 0 \\
\hline 79 & 20.5 & 0.2 & 0.1 & 1.3 & 1.6 & 17.2 & 16.1 & 90 & 75 & 16 & 120 & 0 \\
\hline 82 & 17.0 & 0.1 & 0 & 1.5 & 1.6 & & & & & 170 & & \pm \\
\hline $\begin{array}{l}85 \\
88\end{array}$ & $\begin{array}{l}12.0 \\
19.0\end{array}$ & $\begin{array}{l}0.5 \\
0.1\end{array}$ & 0.2 & 2.3 & 3.0 & 13.9 & $\begin{array}{l}17.7 \\
16.3\end{array}$ & $\begin{array}{l}74 \\
65\end{array}$ & $\begin{array}{l}27 \\
50\end{array}$ & $\begin{array}{l}140 \\
160\end{array}$ & $\begin{array}{r}38 \\
116\end{array}$ & $\stackrel{+}{0}$ \\
\hline $\begin{array}{l}\circ 0 \\
91\end{array}$ & $\begin{array}{l}19.0 \\
15.0\end{array}$ & $\begin{array}{l}0.1 \\
0.3\end{array}$ & 0.2 & 2.0 & $\begin{array}{l}2.5 \\
2.5\end{array}$ & 13.8 & $\begin{array}{l}10.3 \\
15.2\end{array}$ & 41 & 60 & $\begin{array}{l}100 \\
160\end{array}$ & 120 & 0 \\
\hline 94 & 32.5 & 0.7 & 0.1 & 1.7 & 2.5 & 12.4 & 16.7 & 32 & 73 & 165 & 94 & 0 \\
\hline 97 & 18.0 & 0.2 & 0.2 & 3.0 & 3.4 & 13.2 & 12.1 & 92 & 54 & 155 & 157 & 0 \\
\hline
\end{tabular}

to result in a somewhat higher average blood loss in the latter type of experiments. In spite of this difference, our results in both the operated and control series are quite comparable to most values obtained by other investigators for the amounts of blood loss necessary for the production of shock in anethetized dogs (33 to 41 ). The relatively high incidence of shock (35 per cent) in dogs with a total fluid loss of from 2 to 3 per cent of the body weight is not remarkable in view of the repeated observations of many authors that some dogs are unusually susceptible to hemorrhage. Thus shock has been produced by bleeding dogs from 1 to 2.2 per cent of body weight (34, $35,37,39)$.

TABLE III

Incidence of shock with graded vascular fluid loss

\begin{tabular}{l|c|c|c|c}
\hline \hline \multirow{2}{*}{ Total vascular fluid loss } & \multicolumn{3}{|c}{ Incidence of shock } \\
\cline { 2 - 5 } & \multicolumn{2}{|c|}{ With muscle-tie } & \multicolumn{2}{|c}{ Control } \\
\cline { 2 - 5 } & & per cent & & per cent \\
per cent of body weight & $5 / 14$ & 36 & $4 / 13$ & 31 \\
Less than 3.0 & $12 / 17$ & 71 & $2 / 3$ & 67 \\
3.1 to 4.0 & $7 / 9$ & 78 & $3 / 3$ & 100 \\
4.1 to 5.0 & $1 / 1$ & 100 & $4 / 4$ & 100 \\
Greater than 5.0 & & & & \\
\hline
\end{tabular}

There is, therefore, no evidence that under these experimental conditions any agent derived from ischemic tissues is acting generally either to enhance the severity of the shock state directly, or to sensitize the animal to the noxious effects of vascular fluid loss.

Before drawing any general conclusion, however, it is necessary to note the limitations of these experiments. (1) No effort was made to control possible neurogenic stimuli from the traumatized areas except that which is incident to the use of barbiturate anesthesia. However, any consequences of nervous stimuli should have resulted in a difference between the two series of animals. (2) It is possible that too small a mass of tissue was rendered ischemic, and that had such damage been greater in extent, noxious influences might have revealed themselves. (3) In these experiments, ischemia with only a relatively minor degree of actual trauma was produced. It might well be that similar experiments in combination with severe traumatization would reveal the presence of toxic substances derived from the traumatized and ischemic area. Indeed a recent study (24) furnishes evidence suggesting that this may be so. (4) Finally, it is possible that the bleeding 
in these studies may have been too rapid, and that the experiments were not prolonged sufficiently to bring out more subtle differences in susceptibility to vascular fluid loss. Nevertheless, these experiments fail to demonstrate the presence in the general circulation of any noxious substance liberated from ischemic muscle, which accentuates the appearance of shock. Local effects which might influence the amount of traumatic effusion cannot be analyzed in these observations.

\section{SUMMARY}

A comparison of the incidence of shock caused by graded amounts of vascular fluid loss has been made in animals subjected to muscle ischemia and in a series of normal controls. By this technique, no evidence has been obtained for the production in an ischemic region of any agent capable of lowering the general resistance of the animal to the loss of vascular fluid. Following a period of ischemia to the triceps surae muscle groups, there was a loss of fluid into the legs, averaging 1.6 per cent of the body weight. The evidence indicates, therefore, that the important shockproducing factor in muscle anoxia results from influences which increase the local loss of vascular fluid without producing a generalized toxic action. Bibliography follows Paper VI of this series. 